\title{
La capacitación formal en areas sociales para la inserción de un conjunto habitacional social
}

Formal training in social areas for the insertion of a social housing complex

Treinamento formal em áreas sociais para a inserção de um conjunto habitacional social

\section{Garcés Vargas Juan}

Master, Universidad de Guayaquil, Guayaquil, Ecuador, https://orcid.org/0000-0002-9320-2589, juangarcesvargas@yahoo.com

\section{Villao Rodríguez Karina}

Master, Universidad de Guayaquil, Guayaquil, Ecuador, https://orcid.org/0000-0001-9660-4623, kary_alexa@yahoo.com

\footnotetext{
Recibido 22 julio 2019 - Aceptado 30 noviembre 2019

Formación docente - revista iberoamericana de educación

http://www.revista-iberoamericana.org/index.php/es/index

https://creativecommons.org/licenses/by/4.0/deed.es e-ISSN: 2737-632X

Vol - 3 No. 1, enero - marzo 2020

Pags 13-33
}

Resumen La presente intención se basa en realizar un artículo que busca: “optimizar la calidad de vida de la población guayaquileña”, mediante: “CONJUNTO HABITACIONAL SOCIAL "PUERTO PALMAR" EN LA CIUDAD DE GUAYAQUIL", en donde se emplea como objetivo fundamental: "Garantizar el acceso a una vivienda apropiada, segura y digna"; entre las resoluciones, involucra "Promover la organización, responsabilidad familiar y comunitaria, para garantizar su participación en la obtención de residencia nueva o reformada, en el sostenimiento oportuno y adecuado de las viviendas, así como de sus entornos, para así evitar el detrimento y los consecuentes peligros". A partir de la premisa, se arroja un análisis de tipo representativo que beneficiará al proponente para obtener el conocimiento, en zonas aledañas interesadas en hogares de interés social, con 
la finalidad de conocer la idea de comercialización de casas en "PUERTO PALMAR”.

Palabras clave: Desarrollo Urbano, conjunto habitacional, vivienda y vivienda de interés social.

Abstract. The present intention is based on carrying out an article that seeks to: "optimize the quality of life of the Guayaquil population", through: “CONJUNTO HABITACIONAL SOCIAL" PUERTO PALMAR ”IN THE CITY OF GUAYAQUIL", where it is used as a fundamental objective: “ Guarantee access to appropriate, safe and dignified housing "; Among the resolutions, it involves "Promoting the organization, family and community responsibility, to guarantee their participation in obtaining a new or reformed residence, in the timely and adequate support of the homes, as well as their surroundings, in order to avoid detriment and the consequent dangers ". From the premise, a representative analysis is carried out that will benefit the proponent to obtain knowledge, in neighboring areas interested in homes of social interest, in order to know the idea of marketing houses in "PUERTO PALMAR".

Key words: Urban Development, housing complex, housing and low-income housing.

Resumo A presente intenção baseia-se na realização de um artigo que busca: “otimizar a qualidade de vida da população de Guayaquil”, por meio de: "SOCIAL CONJUNTO HABITACIONAL" PUERTO PALMAR "NA CIDADE DE GUAYAQUIL", onde é utilizado como objetivo fundamental: “ Garantir acesso a moradias apropriadas, seguras e dignas ”; Dentre as resoluções, envolve "Promover a responsabilidade da organização, da família 
e da comunidade, para garantir sua participação na obtenção de uma residência nova ou reformada, no apoio oportuno e adequado das casas e do entorno, a fim de evitar prejuízos e os perigos consequentes ". A partir da premissa, é realizada uma análise representativa que beneficiará o proponente a obter conhecimento, em áreas vizinhas interessadas em residências de interesse social, a fim de conhecer a idéia das casas de marketing em "PUERTO PALMAR".

Palavras-chave: Desenvolvimento urbano, complexo habitacional, habitação e habitação de baixa renda.

\section{INTRODUCCIÓN}

El conjunto habitacional "Puerto Palmar" se plantea como un gran reto que permitirá obtener las extensiones básicas para la adecuada proyección, el seguimiento y la valoración del Buen Vivir, el método plantea considerar el acceso universal a los “bienes primordiales", constituidos básicamente por: salud, educación, trabajo y hábitat, aspectos notables para alcanzar otros espacios del bienestar y la mejora en la calidad de la vida. Además de la satisfacción de las necesidades básicas, se considera de vital importancia, también la "extensión del tiempo destinado a vivir en integridad y el abastecimiento de bienes relacionales (afecto, amor, solidaridad, cohesión social)", como caminos para lograr el bienestar y la realización Humana (Plan Nacional para el Buen Vivir 2013-2017).

Los datos del censo de población y vivienda INEC del año 2010, exteriorizaron que el $86 \%$ de la demanda en vivienda se encuentra en los sectores urbanos marginales. Hace varios años atrás, los proyectos habitacionales, eran exclusivamente para los sectores de clase media y alta, actualmente, con la tecnología de materiales prefabricados, es posible que los 
sectores populares también sean considerados dentro de este nicho inmobiliario. Para el año 2010, la tasa anual media de incremento de la urbe fue del $1.58 \%$ con un total de 2.440 .553 de habitantes. (VII Censo de Población y VI de Vivienda, 2010) (VI Censo de Población y V de Vivienda, 2011)

Con este porcentaje, se demuestra que Guayaquil es la ciudad más poblada del Ecuador. A continuación se presenta la proyección de la metrópoli hasta el 2020.

\section{GUAYAQUIL}

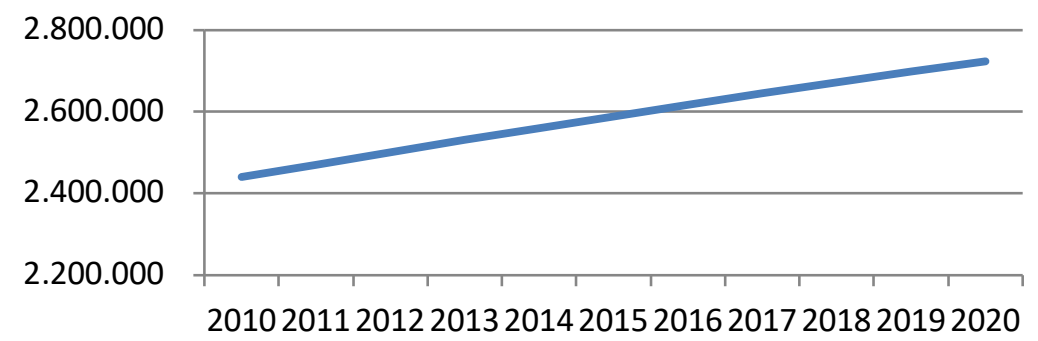

Gráfico 1 Proyección demográfica de la ciudad de Guayaquil

Los indicadores estadísticos con respecto al total de personas ocupadas, subrayan: la edad media de la población (29.1), el porcentaje de personas con cédula de identidad (76.6), el porcentaje de personas con seguro de salud general (36) y el porcentaje de personas que acceden al seguro de salud privado (11.4).

Otros indicadores de igual manera, lo constituyen: la distribución de la población en Guayaquil: 66.5\%, aporte a la ciudad en el 2001 y 64.5\% en el 2010; de esta manera, la diferenciación es mínima. La noción que se cataloga como "El Gran Guayaquil", es una imagen urbanística que tiene el M.I. Municipio de la ciudad en mención, ante el acrecentamiento inmobiliario que da cabida a las clases sociales que cobija la metrópoli. 
Los lugares conocidos como: vía a la Costa, vía a Daule y la autopista Terminal Terrestre-Pascuales, componen los principales puntos de desarrollo en el casco urbano; se espera un futuro encuentro entre los cantones vecinos: Samborondón, Durán, y Daule. En nuestro país, existe un persistente movimiento en lo que respecta a la obtención de viviendas, consecuencia de las políticas aplicadas por el Gobierno en curso; lo que incita que poco a poco el comercio se organice y que las constructoras diseñen planes con interés social, ya que visualizan que es una fuente de negocios importantes.

En la segunda ciudad más grande del Ecuador, varios proyectos inmobiliarios, desean abarrotar el mercado que se aproxima a 80.000 nuevas residencias anuales.

Durante el año 200 se dio una gran ampliación en la fabricación de viviendas, pero en el área metropolitana representaba solo el 43.45\%. En el 2010 las cifras, demuestran que el 60\% de la manufactura total es en el área residencial. Según los valores declarados por los partícipes en el sector de la construcción, éstas coinciden que el 60\% de la intervención de la construcción es en espacios urbanos.

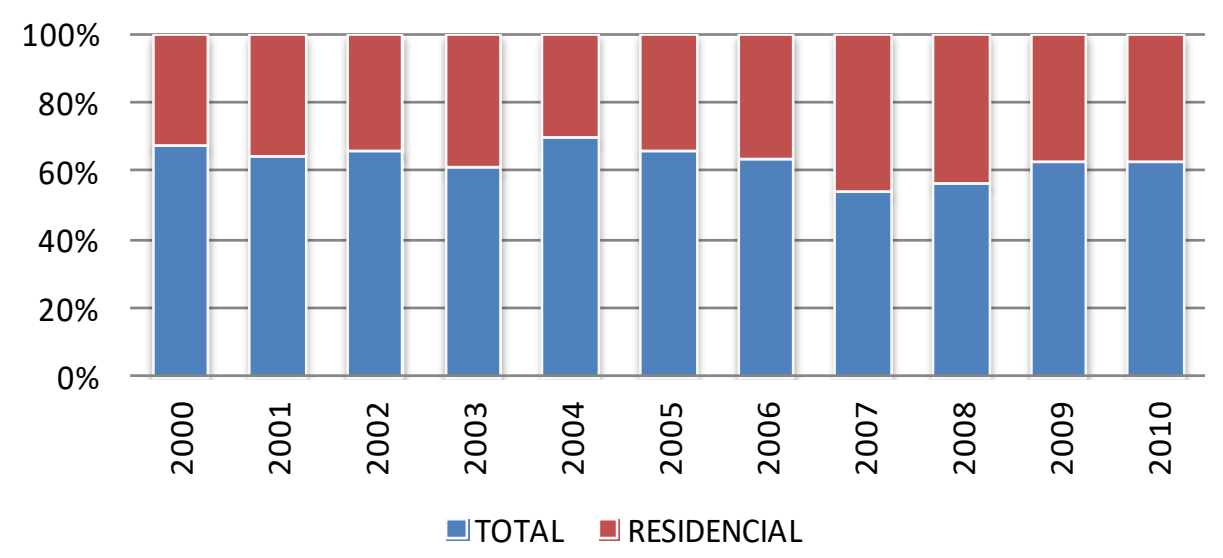

Gráfico 2 Edificaciones por valor declarado en Guayaquil 
La proposición de recursos inmobiliarios de tipo popular, en Guayaquil, no ha poseído mayor interés por parte de los constructores, por ejemplo, "Ciudad Victoria", con 8000 soluciones habitacionales; "Socio Vivienda Guayaquil”, conformado por 12 firmas constructoras guayaquileñas que presagia construir 14000 viviendas (hasta el año 2013); el plan habitacional del Gobierno, "Socio Vivienda Uno", con 2500 hogares para familias de escasos recursos económicos y "Ciudad Olmedo", con 10000 viviendas. La progresión acelerada de la población en Guayaquil, ha intensificado la oferta y la demanda de la vivienda, especialmente en el área metropolitana. Según el censo del 2010, del Instituto Ecuatoriano de Estadística y Censos (INEC), en Guayaquil era de alrededor de 26 mil casas (familias que alquilaban) y aumentaban otras 18 mil familias que subsistían en ranchos y covachas. MIDUVI presenta unas cifras que refieren que la carencia de viviendas nuevas en el 2006 era de 23,3\% y en el 2010 de 19,3\%. Esta cifra está relacionada con las condiciones de miseria, el poder adquisitivo de la población y las fuentes de subvención.

\section{MATERIALES Y MÉTODOS}

En cuanto al déficit cuantitativo, el Ministerio de Desarrollo Urbano y Vivienda y la Subsecretaría de Hábitat y Vivienda, coinciden en que la carencia habitacional bajó el 9\%, pasando de 756806 unidades en el 2006 a 692216 unidades en el 2010. Una verificación general, muestra que las zonas con mayor insuficiencia habitacional cualitativa son: Guayaquil, Machala, Cuenca, Ambato, Quito, Manabí, Los Ríos y la Sierra Centro, pues si bien, las personas tienen un domicilio, son: de caña en la Costa o de adobe en la Sierra. Se diseñó una encuesta a los potenciales interesados en viviendas de 
interés social, con la finalidad de aprobar la idea de comercialización de casas en PUERTO PALMAR

\section{Tabla 1}

Población y muestra

\begin{tabular}{lcc|}
\multicolumn{1}{c}{ Elementos } & Ni (Población) & n (muestra) \\
\hline Habitantes de zonas (jefes y jefas de hogar) & 6500 & 399 \\
aledañas al sector beneficiado & & \\
\end{tabular}

El conocimiento que se tiene, de acuerdo al estudio de tipo descriptivo que se ejecutó en zonas aledañas es la siguiente. Se realizaron 399 encuestas.

Al no realizarse una hipótesis, se considera la probabilidad de ocurrencia de un 50\% y el margen de error de un 5\%; por tal razón y al tratarse de una población finita, el valor de la muestra es de 399.

\section{RESULTADOS}

Al no realizarse una hipótesis, se considera la probabilidad de ocurrencia de un 50\% y el margen de error de un 5\%; por tal razón y al tratarse de una población finita, el valor de la muestra es de 399.

Las conclusiones obtenidas para el tema específico fueron los siguientes:

\section{Tabla 2}

Edad

EDAD

\begin{tabular}{c|lcc} 
ÍTEMS & RANGO & $\begin{array}{c}\text { Valores } \\
\text { absolutos }\end{array}$ & $\%$ \\
1 & $<24$ & 100 & $\mathbf{2 5 \%}$ \\
& $24-34$ & 199 & $\mathbf{5 0 \%}$ \\
& $<34$ & 100 & $\mathbf{2 5 \%}$ \\
& TOTAL & 399 & $\mathbf{1 0 0 \%}$
\end{tabular}


Los resultados obtenidos demuestran que el 50\% de los indagados, en este caso, jefes/as de hogar están en un rango de edad de 24 a 34 años, esto se puede demostrar que existen personas sin acceso a viviendas casadas a temprana edad.

\section{Tabla 3}

Sexo

\begin{tabular}{c|ccc}
\multicolumn{3}{c}{ SEXO } \\
\hline ÍTEMS & Género & $\begin{array}{c}\text { Valores } \\
\text { absolutos }\end{array}$ & $\%$ \\
2 & Masculino & 250 & $63 \%$ \\
& Femenino & 149 & $37 \%$ \\
& TOTAL & 399 & $100 \%$
\end{tabular}

El efecto alcanzado arroja que el $63 \%$ de los encuestados son hombres a diferencia del $37 \%$ que son mujeres. Los escrutinios, en gran parte no lograron obtener equidad de género, debido a que las personas que participaron se negaron a contestar.

\section{Tabla 4}

Viviendas de interés social

\section{CONOCE ACERCA DE LAS VIVIENDAS DE INTERÉS SOCIAL}

\begin{tabular}{|c|c|c|c|}
\hline ÍTEMS & & $\begin{array}{c}\text { Valores } \\
\text { absolutos }\end{array}$ & $\%$ \\
\hline \multirow[t]{3}{*}{3} & $\mathrm{Si}$ & 145 & $36 \%$ \\
\hline & No & 254 & $64 \%$ \\
\hline & TOTAL & 399 & $100 \%$ \\
\hline
\end{tabular}

En referencia a la locución: "moradas de interés social", se pudo comprobar que las personas encuestadas, no conocen esta terminología, por entrevistas realizadas, estas, se enfocan en la expresión "viviendas de tipo popular", no obstante, ambas poseen la misma relación. 


\section{Tabla 5}

Casa propia

TIENE USTED CASA PROPIA

\begin{tabular}{c|lcc} 
ÍTEMS & \multicolumn{3}{|c}{$\begin{array}{c}\text { Valores } \\
\text { absolutos }\end{array}$} \\
4 & SI & 12 & \% \\
& NO & 150 & $3 \% \%$ \\
& NO RESPONDE & 237 & $59 \%$ \\
& TOTAL & 399 & $100 \%$
\end{tabular}

Cuando se refiere a la existencia de residencia propia, se evidenció que el $38 \%$ carece de vivienda a diferencia del 3\% que manifiesta si tener hogar establecido, hubo un $59 \%$ de personas que no respondían probablemente por temores.

\section{Tabla 6}

Espacios

¿El espacio en donde vive ahora no es suficiente para la cantidad de personas que integran su familia?

\begin{tabular}{|c|c|c|c|}
\hline ÍTEMS & & $\begin{array}{c}\text { Valores } \\
\text { absolutos }\end{array}$ & $\%$ \\
\hline \multirow[t]{3}{*}{5} & $\mathrm{Si}$ & 340 & $85 \%$ \\
\hline & No & 59 & $15 \%$ \\
\hline & TOTAL & 399 & $100 \%$ \\
\hline
\end{tabular}

Con respecto al hacinamiento en los sectores populares por parte de las familias guayaquileñas, el $85 \%$ manifiesta que efectivamente viven aglomeradamente, seguido del 15\% que manifiesta que el espacio es el adecuado. 
Formación docente

revista iberoamericana de educación

\section{Tabla 7}

Casas

¿Ha pensado usted en el último año, en obtener una casa para darle una mejor calidad de vida a su familia?

\begin{tabular}{c|lcc}
\hline ÍTEMS & \multicolumn{3}{|c}{$\begin{array}{c}\text { Valores } \\
\text { absolutos }\end{array}$} \\
\multirow{2}{*}{6} & $\mathrm{Si}$ & 250 & $63 \%$ \\
& $\mathrm{No}$ & 149 & $37 \%$ \\
& TOTAL & 399 & $100 \%$
\end{tabular}

Referente a la información mostrada, el 63\% considera que si ha analizado en el último año adquirir una casa para darle un mejor estilo vida a su familia, esto se puede validar con la demanda actual de viviendas, el mercado habitacional ha tenido un despunte significativo en los últimos años.

\section{Tabla 8}

Vivienda

¿Usted considera que las unidades básicas de vivienda ofertadas a la población en la actualidad, cubren con los aspectos básicos en cuanto a su estética, funcionalidad, seguridad y valor monetario?

\begin{tabular}{c|lcc}
\hline ÍTEMS & \multicolumn{3}{|c}{$\begin{array}{c}\text { Valores } \\
\text { absolutos }\end{array}$} \\
7 & $\mathrm{Si}$ & 256 & $\% 4 \%$ \\
& No & 143 & $36 \%$ \\
& TOTAL & 399 & $100 \%$
\end{tabular}

Muestra que el $65 \%$ asegura que las viviendas ofertadas al público en la actualidad, cubren con los aspectos básicos en cuanto; a su estética, funcionalidad, seguridad y precio, no obstante, se deben de considerar aspectos como los de acceso a crédito que también han sido comprobados. 


\section{Tabla 9}

Trámites

¿Según su opinión, son factibles los trámites para la obtención de una vivienda 0 un crédito para vivienda?

\begin{tabular}{c|lcc}
\hline ÍTEMS & \multicolumn{3}{|c}{$\begin{array}{c}\text { Valores } \\
\text { absolutos }\end{array}$} \\
$\mathbf{8}$ & Si & 45 & $11 \%$ \\
& No & 354 & $89 \%$ \\
& TOTAL & 399 & $100 \%$
\end{tabular}

Los cabezas de hogar, manifiestan que existen muchos inconvenientes al momento de realizar las gestiones para la obtención de una vivienda o un crédito para el mismo fin. De acuerdo a los resultados, el 89\% manifiesta que son dificultosos a diferencia de solo un $11 \%$ que manifiesta que son posibles.

\section{Tabla 10}

Unidades de vivienda

¿Prefiere usted adquirir una casa en unidades de vivienda o designaría que se construya en un terreno propio?

\begin{tabular}{c|lcc}
\hline ÍTEMS & \multicolumn{1}{c}{$\begin{array}{c}\text { Valores } \\
\text { absolutos }\end{array}$} & \% \\
\multirow{2}{*}{9} & Unidades de vivienda & 210 & $53 \%$ \\
& terreno propio & 189 & $47 \%$ \\
& TOTAL & 399 & $100 \%$
\end{tabular}

Los datos muestran, que el 53\% manifiesta que elige adquirir en unidades de vivienda a diferencia que se edifique en un terreno propio, esto probablemente debido a las facilidades que obtienen de los conjuntos habitacionales en donde les otorgan servicios básicos eficientes y de distracción para niños y adultos. 


\section{Tabla 11}

Crédito hipotecario

¿Una casa nueva promovida a través de crédito hipotecario de interés social, sería un recurso eficiente para su problema de vivienda? ÍTEMS

10

$\begin{array}{lcc}\text { SI } & \begin{array}{c}\text { Valores } \\ \text { absolutos }\end{array} & \% \\ \text { NO } & 300 & 75 \% \\ \text { No responde } & 54 & 14 \% \\ \text { TOTAL } & 45 & 11 \% \\ & 399 & 100 \%\end{array}$

Los resultados indican, que el $75 \%$ considera que una casa nueva adquirida a partir de un crédito hipotecario de interés social, sería una excelente medida para su inconveniente en la adquisición de una vivienda, a diferencia del 14\% que manifiesta lo contrario, además un $11 \%$ se abstiene de contestar.

Tabla 12

Plan de desarrollo

¿CONSIDERA USTED QUE SE DEBE DE INICIAR UN PLAN DE DESARROLLO URBANÍSTICO DE VIVIENDAS POPULARES, LLAMADA: "PUERTO PALMAR" EN GUAYAQUIL? ÍTEMS

11
Valores absolutos

$\begin{array}{lcc}\text { Si } & 357 & 89 \% \\ \text { No } & 42 & 11 \% \\ \text { TOTAL } & 399 & 100 \%\end{array}$

Esta pregunta es indispensable para así aprobar el trabajo de investigación y de esta manera mostrar los efectos concluyentes. El 89\% del total de los encuestados, manifiesta que si debe emprenderse un plan de desarrollo urbanístico de viviendas de tipo popular "Puerto Palmar" en Guayaquil. 


\section{Tabla 13}

Disposición a pagar

\begin{tabular}{c|ccc}
\multicolumn{2}{c}{ ¿CUÁNTO ESTARÍA DISPUESTO A CANCELAR POR LA } \\
RESIDENCIA? \\
\hline ÍTEMS & \multicolumn{3}{c}{$\begin{array}{c}\text { Valores } \\
\text { absolutos }\end{array}$} \\
\multirow{3}{*}{$\mathbf{1 2}$} & PROMEDIO & 17800 & DÓLARES \\
& DESVIACIÓN & 3400 & DÓLARES
\end{tabular}

Respecto a la disposición a pagar, se pudo evidenciar que de acuerdo al mercado objetivo, el promedio por la cancelación económica de viviendas es de 17800 dólares, el coste es pequeño, debido a que son programas de vivienda de interés social donde los estratos económicos que acceden son de clase media baja.

La metrópoli, necesita amparar la carencia de viviendas en la población urbano marginal, que ha sido la más afectada en lo referente a plan habitacional por el sector constructivo en la costa. Existen intenciones de edificaciones de tipo social con crédito autorizado. De esta manera albergarían la esperanza del sector medio bajo de obtener vivienda propia. $\mathrm{Si}$ no nos percatamos de esta demanda, albergaríamos la posibilidad que acreciente las invasiones de tierra, originando mayor desorganización territorial.

Debido a las pocas posibilidades de crédito financiero, la clase baja termina siendo descalificada al nivel de ofertas de viviendas, ya que los planes habitacionales, generalmente agrupan sus inversiones en el sector social de clase media-alta; por esta razón, el sector financiero como lo son: bancos, cooperativas y mutualistas, no buscan arriesgarse otorgando créditos a personas que carecen de beneficios económicos. 
Para acceder a un crédito hipotecario, la situación se vuelve compleja, ya que la cuota inicial para adquirir una residencia, generalmente es del $30 \%$, ya que ese porcentaje es el que solicitan las entidades financieras para acceder a ese anticipo; a esto sumada la situación económica que vive el país, le resulta muy difícil poseer dicha cantidad, las constructoras dan facilidades para el pago de la entrada de hasta 24 meses de plazo, no obstante, una vez cancelado esa cuota, es responsabilidad del interesado, conseguir el préstamo con alguna entidad financiera. En la actualidad, la mayoría de los propósitos privados de urbanización, están enfocados a las clases media y alta de la población por su objeto natural de la obtención de mayores dividendos. Por otro lado, las entidades financieras del país como bancos y cooperativas, facilitan préstamos de vivienda, teniendo como base la aportación del $30 \%$ por parte del usuario, así como de un estudio íntegro respecto a la base económica del implicado, bienes y/o patrimonios cruzados con el endeudamiento actual. De acuerdo al estudio realizado, el sector medio bajo, aspira a viviendas que oscilan entre los UDS $15,000.00$ y USD 25,000.00 con circunstancias de pago factibles, contribuyendo un monto inicial una entrada de hasta el 30\%. Las transformaciones anheladas a partir de la ejecución del proyecto, es favorecer las condiciones de habitar de las personas que adquieran uno de estos domicilios. El sector constructor en conjunto con la parte prestamista, alternen sus recursos en este tipo de proyectos que son de mucha rentabilidad y socialmente fructuosos. Así mismo, se aguarda generar fuentes de trabajo y acortar en gran medida las invasiones de terrenos privados o municipales. Con el proyecto de urbanización de viviendas populares "PUERTO PALMAR", se reviste una mínima parte la demanda actual en la ciudad de Guayaquil, sin embargo, este plan habitacional, favorecerá para que inversionistas privados y/o públicos se enfoquen en los planes urbanísticos de estratos sociales medios bajos y no solo para la clase alta. 
Durante el desarrollo del análisis, posiblemente se hayan encontrado nuevas necesidades de inversión o excluido otras previstas inicialmente. En el plan de inversión, es el momento de especificar qué tipo de inversiones son indispensables, cuáles tienden a ser superfluas o de aplazamiento, siempre teniendo en cuenta que se debe alcanzar la mínima inversión para que la acción sea operativa.

Cuando citamos los valores a invertir, se estima establecer un préstamo, en la tabla 11, se proyecta el monto de la inversión total, en donde muestra la cuantía y la forma en que se asigna el capital para promover la sociedad y el desarrollo de la actividad empresarial, hasta alcanzar el umbral de rentabilidad. Se aprecia un total de 100 viviendas a concederse en el total de trimestres de estimación del plan de viviendas.

\section{Tabla 15}

Inversión Inicial

\section{Inversión Total}

\begin{tabular}{r|cr} 
INVERSIÓN FIJA & $\$$ & $44.487,20$ \\
GASTOS PRE-OPERACIONALES & $\$$ & $9.223,08$ \\
CAPITAL DE TRABAJO & $\$ 171.244,49$ \\
TOTAL & $\$$ & $224.954,77$
\end{tabular}

Para cerciorarse del valor obtenido en el capital de trabajo, se estimó un período de diferencia de tres meses, reflexionando en los costos de producción, administrativos y comercialización. Las contribuciones de los socios, serán el capital propio aportado por los integrantes de la sociedad y que conforma el capital, no es reembolsable, contando con el valor de US\$ 100.000,00 dólares; este concepto indica el volumen de financiación propia; y la parte restante, con el monto de US\$ 124.954,77 dólares; se la conseguirá 
mediante un préstamo bancario. Para considerar el VAN y el TIR, se necesita realizar un estudio de ambos en donde se debe precisar la tasa de descuento, la cual se la expresa como el valor que se debe de cancelar por los fondos solicitados para capitalizar la inversión, lo que representa una medida de rentabilidad nimia que se exigirá al proyecto de acuerdo a su compromiso.

A continuación, se muestran los flujos de caja netos que dan origen al cálculo del VAN y TIR.

\section{Tabla 16}

Flujo de caja

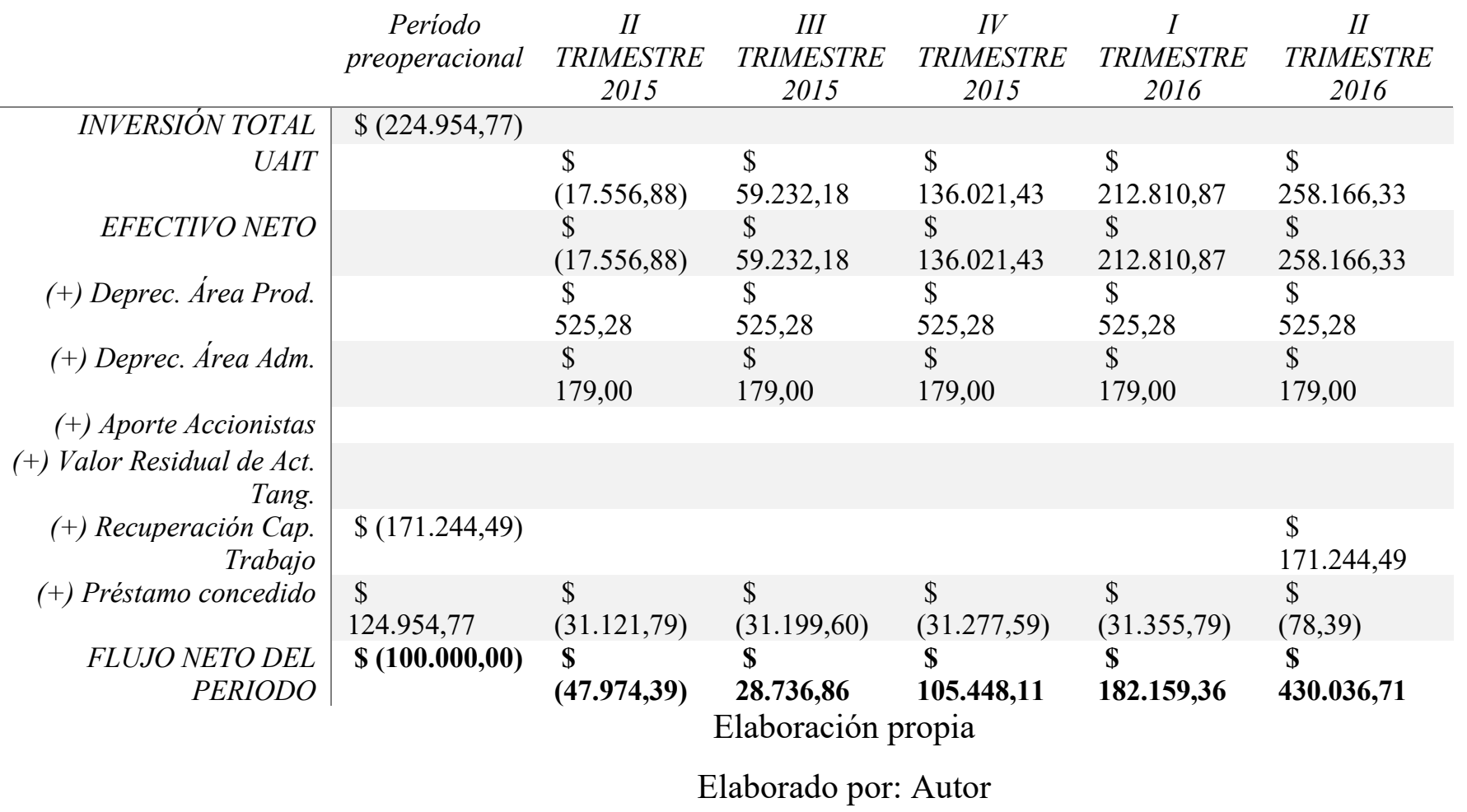

Al revisar el flujo de caja, reconocemos que los fundamentos logrados, alcanzan una tasa interna de retorno social que termina siendo muy atractiva, reflejando un valor de 43,48 \% y un VAN positivo de $\$ \$ 288.932,57$ 
Así puede finalizarse que el propósito sí genera beneficios económicos, ya que la TIR > tasa de descuento y el VAN es superior a 0 como se detalla a continuación.

Tabla 17

Cálculo del VAN Y TIR

TIR 43,48\%

\begin{tabular}{l|l}
$V A N$ & $\$ 288.932,57$
\end{tabular}

En el momento en que se hayan calculado y proyectado los rubros, se muestra a continuación detallado el valor total de la iniciativa.

Tabla 18

Cálculo del VAN Y TIR

DETALLE DESCRIPCIÓN

\begin{tabular}{r|cr} 
COSTOS DE PRODUCCIÓN & $\$ 897.242,94$ \\
GASTOS ADMINISTRATIVOS Y DE VENTAS & $\$$ & $133.111,50$ \\
INVERSIÓN INICIAL & $\$ 155603,7697$ \\
TOTAL VALOR DEL PROYECTO & $\$ 1.185 .958,21$
\end{tabular}

Para los contextos, se consideró a las variables "precio de las residencias" para las simulaciones, es así como se detalla a continuación: 


\section{Tabla 19}

Escenarios

\begin{tabular}{|c|c|c|c|}
\hline Escenarios & Variable & & \\
\hline & Precio & $V A N$ & $T I R$ \\
\hline Optimista & $\$ 16.000,00$ & $\$ 356.290,46$ & $50 \%$ \\
\hline Normal & $\$ 15.400,00$ & $\$ 288.932,57$ & $43 \%$ \\
\hline Pesimista & \$ $12.994,91$ & $\$$ & $3 \%$ \\
\hline
\end{tabular}

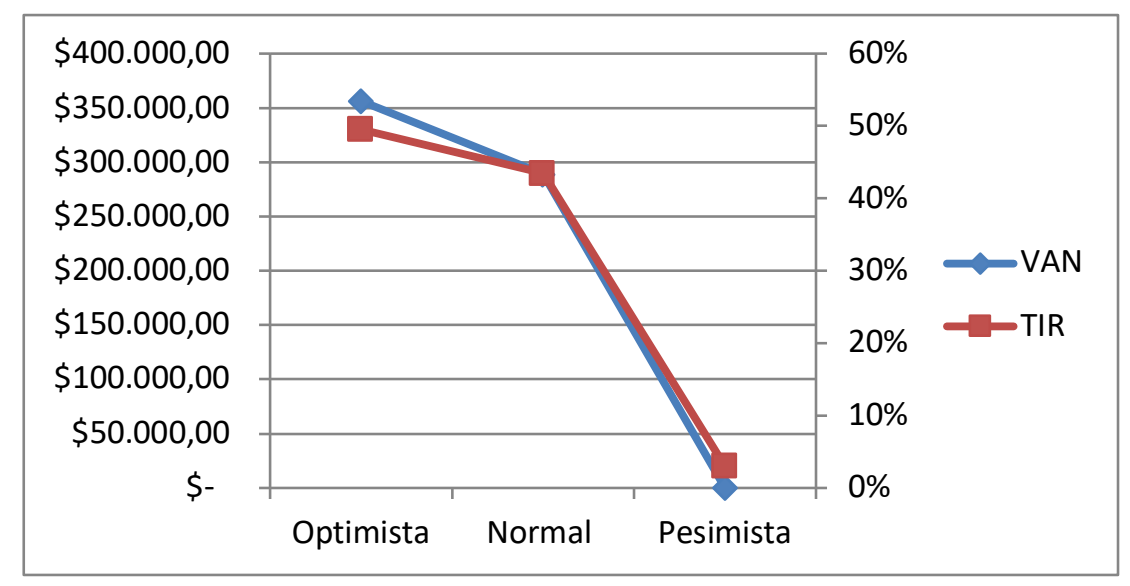

Gráfico 15 Escenarios. Elaboración propia

\section{CONCLUSIONES}

La proposición de un plan de desarrollo residencial, con interés social, manifiesta la demanda existente por este tipo de bienes que ha tenido ampliaciones reveladoras en los últimos años en el país; de igual manera, se pudo afirmar que para el sector a intervenir, existe diversa inclinación y sobre todo, apertura para la adquisición de inmuebles.

Las viviendas de interés social siempre tendrán aceptación; pese a la crisis económica, estas residencias, son de fácil acceso para las personas de clase media y baja. 
Referente al levantamiento de datos realizado a los potenciales beneficiarios, estos denotaron inquietud por los trámites a ejecutar para la obtención de la viviendas, por lo tanto, no se preocupan de manera proporcional por el diseño ni acabados, más bien, buscan cubrir sus necesidades básicas.

Se consideró la rentabilidad desde el punto de vista financiero, tanto el VAN como la TIR validaron el hecho que el plan habitacional sea conveniente, debe ser desarrollado y la inversión cometida se recuperará bajo el supuesto que con una buena campaña de comercialización y venta en los 5 trimestres se puedan ofrecer las 100 casas.

\section{REFERENCIAS}

ARANDA, Alcides. Planificación estratégica educativa. Segunda edición. Ediciones Abya - Yala. Quito - Ecuador 2007.

AULESTIA, Diego, Medios de vida urbanos y vivienda en Ecuador, Quito: FLACSO Ecuador, 2007

BONDUKI, Nabil, Política de vivienda e inclusión social en Brasil: revisión histórica y nuevas perspectivas en el gobierno de Lula. Quito, FLACSO Ecuador, 2007

BERNAL, César. Metodología de la investigación. Segunda edición. Editoriales Pearson - Prentice Hall. México 2006.

BESLEY, S. y Brigham, E. F. (2005). Fundamentos de administración financiera. 12a. ed. México: McGraw-Hill

BREALEY, R. y Myers, S. (1992). Principios de finanzas corporativas. 3a. ed. Caracas: McGraw-Hill.

CELY, Nathalie. HERNÁNDEZ, Iván. Redes de competitividad y productividad compartida. Quito - Ecuador 2003.

DEDE, Graciela, El derecho a la vivienda adecuada. Instituto del Tercer Mundo, 2007 
HERNÁNDEZ, Roberto. SAMPIERI, Carlos. BAPTISTA, Pilar.

Metodología de la investigación. Editorial Mc. Graw-Hill. México 2003.

MÉNDEZ, Carlos. Metodología, diseño y desarrollo del proceso de investigación con énfasis en ciencias empresariales. 4ta edición.

Editorial Limusa. Bogotá - Colombia 2006.

PACHECO, Oswaldo. Investigación I. Primera Edición. Editorial

Mundiciencia. Guayaquil - Ecuador 2000.

PACHECO, Oswaldo. Proyectos Educativos. Editorial Mundiciencia, Guayaquil - Ecuador 2000.

Organización Panamericana de la Salud (OPS): Vivienda y Asentamientos humanos. Vivienda rural y urbana. Marco socio histórico.

Rodríguez Morales, A., Barros Bastida, C., \& Milanés Gómez, R. (2019).

Profesionalización docente y formación desde un nuevo currículo en la Universidad de Guayaquil. Revista Universidad y Sociedad,11(1), 243-248.

Tapia-León, M., Rivera Villalta, M. D. C., Luján-Mora, S., \& Barros Bastidas, C. I. (2017). Análisis de la calidad de los resúmenes de tesis de grado de las universidades del Ecuador respecto a normas internacionales.

von Feigenblatt, Otto Federico, The Importance of Culture in Emic Interpretations of the History of Thailand's Southern Separatist Movement: The 'Gentlemen's Agreement' of 1943 and the Relationship of Malaysia with the Separatists (October 9, 2009). Ritsumeikan Center for Asia Pacific Studies Working Paper No. 097 , Available

at SSRN: https://ssrn.com/abstract=1486224 or http://dx.doi.org/10.213 9/ssrn.1486224 
von Feigenblatt, Otto Federico, Myanmar (Burma): Nationalism and International Ideology Trumps Emic Norms of Interaction and Governance (August 12, 2015). International Journal of Humanities and Social Science Review, 1(1), 20-24, December 2014, Available at SSRN: https://ssrn.com/abstract $=2706854$

von Feigenblatt, Otto Federico, The Human Security Theory of Integrative Negotiation for Sociopolitical Conflicts: The Thai Color Divide as a Case Study. Asia Pacific World, Vol. 1, No. 2, pp. 61-82, 2010, Available at SSRN: https://ssrn.com/abstract $=1722268$ 